\title{
Nitrate reductase assay for detection of drug resistance in Mycobacterium tuberculosis: simple and inexpensive method for low-resource laboratories
}

\section{Correspondence \\ Ernesto Montoro \\ emontoro@ipk.sld.cu}

Received 24 January 2006

Accepted 26 February 2006

\author{
Dihadenys Lemus, ${ }^{1}$ Ernesto Montoro, ${ }^{1}$ Miguel Echemendía, ${ }^{1}$ \\ Anandi Martin, ${ }^{2}$ Françoise Portaels ${ }^{2}$ and Juan Carlos Palomino ${ }^{2}$ \\ ${ }^{1}$ Instituto de Medicina Tropical 'Pedro Kouri', Autopista Novia del Mediodía Km 61/2, \\ Apartado Postal 601, La Lisa, La Habana, Cuba \\ ${ }^{2}$ Institute of Tropical Medicine, Nationalestraat 155, 2000 Antwerp, Belgium
}

The nitrate reductase assay (NRA) was used as an alternative method for detection of resistance to the first-line antituberculous drugs isoniazid, rifampicin, ethambutol and streptomycin. A total of 320 strains of Mycobacterium tuberculosis were studied and the results compared with the proportion method (PM) on Löwenstein-Jensen medium. The mean time to obtain results was 10 days and the overall agreement between the NRA and PM was 98.8\%. The NRA was easy to perform and represents a useful tool for rapid and accurate determination of drug-resistant M. tuberculosis strains in low-resource countries.

\section{INTRODUCTION}

Tuberculosis (TB) is one of the most important health problems worldwide. The emergence of multidrug-resistant (MDR) strains of Mycobacterium tuberculosis resistant to at least isoniazid (INH) and rifampicin (RMP) has hindered control of this disease. For this reason, the rapid diagnosis of TB drug resistance is a priority to avoid the spread of resistant strains (Musa et al., 2005; Palomino, 2005).

There are different methods for detection of TB drug resistance. The proportion method (PM) and other conventional tests, based on the measurement of growth in culture media containing antibiotics, require several weeks to give results. The BACTEC radiometric system has the advantage of being more rapid (5-10 days), but requires the use of radioisotopes and can be costly to be performed routinely. Commercial tests (MGIT, ETest) and molecular tools (INNO-LiPA) have been proposed, but are expensive and also impractical for routine use (Lemus et al., 2004; Palomino, 2005).

For developing countries, it would be useful to have a simple and inexpensive test that could rapidly detect drug-resistant M. tuberculosis strains. Several methods have been reported, including colorimetric methods that use redox indicators (MTT and resazurin) and phage amplification technology (Abate et al., 1998; Martin et al., 2003; Simboli et al., 2005).

Abbreviations: EMB, ethambutol; $I N H$, isoniazid; MDR, multidrugresistant; NRA, nitrate reductase assay; PM, proportion method; RMP, rifampicin; STR, streptomycin.
An alternative method is the nitrate reductase assay (NRA), previously reported as a useful tool for rapid and accurate detection of resistance to first-line antituberculous drugs (Ängeby et al., 2002; Panaiotov \& Kantardjiev, 2002; Coban et al., 2004; Montoro et al., 2005).

The objective of this research was to evaluate the performance of the NRA as a simple and rapid alternative method for routine detection of resistance to first-line antituberculous drugs. The NRA uses colorimetric detection of nitrite as an indication of growth for drug-susceptibility testing.

\section{METHODS}

Mycobacterial strains. A total of 320 strains of M. tuberculosis were studied. Of these, 235 were referred to the National Reference Tuberculosis Laboratory of the Instituto de Medicina Tropical 'Pedro Kouri' for drug-susceptibility testing between October 2004 and July 2005; the other 85 strains belonged to the collection of this laboratory. M. tuberculosis reference strains H37Rv (ATCC 27294) sensitive to first-line antituberculous drugs, RMP-resistant (ATCC 35838), INH-resistant (ATCC 35822), ethambutol (EMB)-resistant (ATCC 35837) and streptomycin (STR)-resistant (ATCC 35820) were used as susceptible and resistant controls. All strains were subcultured in Löwenstein-Jensen (LJ) medium for 4 weeks before being studied.

Antituberculous drugs. INH, STR, EMB and RMP (SigmaAldrich) were each prepared at a concentration of $1 \mathrm{mg} \mathrm{ml}^{-1}$ in distilled water with the exception of RMP, which was diluted in methanol. Stock solutions were filter-sterilized and stored at $-70^{\circ} \mathrm{C}$. The evaluated concentrations were $0 \cdot 2,4,2$ and $40 \mu \mathrm{g} \mathrm{ml}^{-1}$ for INH, STR, EMB and RMP, respectively. 
Proportion method. The PM was performed on LJ medium according to the standard procedure with the recommended critical concentration (Canetti et al., 1969).

Nitrate reductase assay. The NRA was performed according to the protocol described previously by Ängeby et al. (2002). The inoculum turbidity was adjusted to a McFarland tube no. 1 and diluted 1:10 in PBS. Undiluted suspension $(200 \mu \mathrm{l})$ was inoculated into tubes of $\mathrm{LJ}$ medium with $\mathrm{KNO}_{3}\left(1 \mathrm{mg} \mathrm{ml}{ }^{-1}\right)$ containing each of the drugs at the concentrations described above and $200 \mu \mathrm{l}$ of the $1: 10$ dilution was inoculated into three control tubes without antibiotics. The assay was developed with reagent mix $(50 \%$ conc. $\mathrm{HCl}$, $0 \cdot 2 \%$ sulfanilamide and $0 \cdot 1 \% \mathrm{~N}$-(1-naphthyl)ethylenediamine dihydrochloride at ratio of $1: 2: 2$ ) after 7 days of incubation at $37^{\circ} \mathrm{C}$ by adding $500 \mu \mathrm{l}$ reagent mix to one control tube. If the clear reagent mix turned pink, the drug-containing tubes were subsequently developed. If there was no colour change, the tubes were reincubated and the procedure repeated on days 10 and 14. An isolate was considered resistant if the drug-containing tube produced a colour change that was more intense than the drug-free tube.

\section{RESULTS AND DISCUSSION}

The contribution of TB laboratories to the management of MDR TB in the world is important for the rapid determination of drug susceptibility, especially in low-resource countries where most cases of MDR TB occur (Montoro et al., 2005). Numerous alternative methods have been proposed for the detection of TB drug resistance. Most have shown high sensitivity and specificity values for INH and RMP, the most important drugs in TB treatment (Ängeby et al., 2002; Lemus et al., 2004; Simboli et al., 2005).

Here, the NRA was used as alternative method for rapid detection of resistance to first-line antituberculous drugs in $320 \mathrm{M}$. tuberculosis strains. The mean time required to obtain results was 10 days.

The results are presented in Table 1. For INH, both methods detected 55 resistant strains and 260 susceptible strains; five false-negative results were obtained with the NRA. For STR, 83 strains were correctly identified as resistant and 232 as susceptible by both methods; five strains gave discordant results, three of which were false negative, being susceptible

Table 1. Drug-susceptibility testing of 320 M. tuberculosis strains by the NRA and PM

\begin{tabular}{|llccc|}
\hline \multirow{2}{*}{ Drug } & NRA & \multicolumn{2}{c}{ Proportion method } & \multirow{2}{*}{$\begin{array}{c}\text { Agreement } \\
\text { (\%) }\end{array}$} \\
\cline { 3 - 4 } & & Resistant & Susceptible & \\
\hline \multirow{2}{*}{ INH } & Resistant & 55 & 0 & $98 \cdot 4$ \\
& Susceptible & 5 & 260 & \\
STR & Resistant & 83 & 2 & $98 \cdot 4$ \\
& Susceptible & 3 & 232 & \\
EMB & Resistant & 22 & 0 & $99 \cdot 1$ \\
& Susceptible & 3 & 295 & \\
RMP & Resistant & 46 & 0 & $99 \cdot 1$ \\
& Susceptible & 3 & 271 & \\
& & & & \\
\hline
\end{tabular}

by the NRA but resistant by the PM. For EMB, 22 true positive (resistant) results were obtained and 295 strains were defined as susceptible by both methods; three strains were misidentified as susceptible by the NRA. For RMP, all of the susceptible strains were correctly identified by the NRA and 46/49 resistant strains were correctly detected by this method.

The sensitivity of the NRA was $91 \cdot 7 \%, 96 \cdot 5 \%, 88 \cdot 0 \%$ and $93.9 \%$ for INH, STR, EMB and RMP, respectively, and the specificity was higher than $99 \cdot 1 \%$ for all of the drugs. The overall agreement between the NRA and PM was $98 \cdot 8 \%$.

In this study, the accuracy with the 'gold standard' PM was very good according to the criteria established by the WHO/ IUATLD Supranational Laboratory Network, which has proposed accuracy levels of $99 \cdot 0 \%$ and $97.0 \%$ for RMP and INH, respectively, and $92.0 \%$ for EMB and STR as reasonable goals for reference laboratories. Although it is well known that STR and EMB are difficult drugs to test, even by conventional standard methods (Laszlo et al., 2002), the results obtained in this study were promising, as few discordant results were obtained.

In a previous study carried out in our laboratory (Montoro et al., 2005), the NRA was compared with colorimetric methods (using MTT and resazurin). Although no statistical difference was found between them, the highest accuracy was for the NRA $(98 \cdot 2 \%)$. Other studies have reported high sensitivity and specificity values for INH and RMP (Ängeby et al., 2002; Coban et al., 2004).

In a multicentre study, the NRA was evaluated in different settings to determine the susceptibility of M. tuberculosis to first-line antituberculosis drugs. The accuracy was higher than $96 \cdot 6 \%$ for INH, EMB and RMP, while that for STR was lower $(85 \cdot 3 \%)$ (Martin et al., 2005a).

The NRA has the potential to be used for the evaluation of second-line drugs. In 2005, Martin et al. (2005b) reported the evaluation of this method for ofloxacin resistance detection and found complete concordance with the agar PM.

Recently, with the aim of reducing the turnaround time for susceptibility results, the NRA was applied directly to sputum smear-positive samples. Musa et al. (2005) reported full concordance for RMP resistance detection, although some discordant results were obtained for other drugs. Similar results have been reported by Solis et al. (2005). The advantage of the direct method is that results are available sooner and better represent the patient's original bacterial population (Ramachandran \& Paramasivan, 2003). Current studies are in progress to evaluate this method in our laboratory.

In conclusion, the NRA constitutes a useful tool for detection of TB drug resistance in low-resource countries with limited laboratory facilities due to its low cost, ease of performance and lack of requirement for sophisticated equipment. 


\section{ACKNOWLEDGEMENTS}

This study was supported by the European Commission RDG (INCODEV Programme), project no. ICA4-CT-2001-10087. These results were presented during the 36th World Conference on Lung Health of the International Union Against Tuberculosis and Lung Disease, 18-22 October 2005, Paris, France.

\section{REFERENCES}

Abate, G., Mshana, R. N. \& Miorner, H. (1998). Evaluation of a colorimetric assay based on 3-(4,5-dimethyl thiazol-2-yl)-2,5-diphenyl tetrazolium bromide (MTT) for rapid detection of rifampicin resistance in Mycobacterium tuberculosis. Int J Tuberc Lung Dis 2, 10111016.

Ängeby, K. A., Klintz, L. \& Hoffner, S. E. (2002). Rapid and inexpensive drug susceptibility testing of Mycobacterium tuberculosis with a nitrate reductase assay. J Clin Microbiol 40, 553-555.

Canetti, G., Fox, W., Khomenko, A. L., Mahler, H. T., Menon, N. K., Mitchison, D. A., Rist, N. \& Smelev, N. A. (1969). Advances in techniques of testing mycobacterial drug sensitivity, and the use of sensitivity tests in tuberculosis control programs. Bull World Health Organ 41, 21-43.

Coban, A. Y., Birinci, A., Ekinci, B. \& Durupinar, B. (2004). Drug susceptibility testing of Mycobacterium tuberculosis with nitrate reductase assay. Int J Antimicrob Agents 24, 304-306.

Laszlo, A., Rahman, M., Espinal, M., Raviglione, M. \& WHO/IUATLD Network of Supranational Reference Laboratories (2002). Quality assurance programme for drug susceptibility testing of Mycobacterium tuberculosis in the WHO/IUATLD Supranational Reference Laboratory Network: five rounds of proficiency testing, 1994-1998. Int J Tuberc Lung Dis 6, 748-756.

Lemus, D., Martin, A., Montoro, E., Portaels, F. \& Palomino, J. C. (2004). Rapid alternative methods for detection of rifampicin resistance in Mycobacterium tuberculosis. J Antimicrob Chemother 54, 130-133.

Martin, A., Camacho, M., Portaels, F. \& Palomino, J. C. (2003). Resazurin microtiter assay plate testing of Mycobacterium tuberculosis susceptibilities to second-line drugs: rapid, simple, and inexpensive method. Antimicrob Agents Chemother 47, 3616-3619.

Martin, A., Montoro, E., Lemus, D. \& 8 other authors (2005a). Multicenter evaluation of the nitrate reductase assay for drug resistance detection of Mycobacterium tuberculosis. J Microbiol Methods 63, 145-150.

Martin, A., Palomino, J. C. \& Portaels, F. (2005b). Rapid detection of ofloxacin resistance in Mycobacterium tuberculosis by two low-cost methods: resazurin and nitrate reductase assay. J Clin Microbiol 43, 1612-1616.

Montoro, E., Lemus, D., Echemendía, M., Martin, A., Portaels, F. \& Palomino, J. C. (2005). Comparative evaluation of the nitrate reduction assay, the MTT test, and the resazurin microtiter assay for drug susceptibility testing of clinical isolates of Mycobacterium tuberculosis. J Antimicrob Chemother 55, 500-505.

Musa, H. R., Ambroggi, M., Souto, A. \& Ängeby, K. A. (2005). Drug susceptibility testing of Mycobacterium tuberculosis by a nitrate reductase assay applied directly on microscopy-positive sputum samples. J Clin Microbiol 43, 3159-3161.

Palomino, J. C. (2005). Nonconventional and new methods in the diagnosis of tuberculosis: feasibility and applicability in the field. Eur Respir J 26, 339-350.

Panaiotov, S. \& Kantardjiev, T. (2002). Nitrate reductase assay for drug susceptibility testing of Mycobacterium tuberculosis. J Clin Microbiol 40, 3881-3882.

Ramachandran, R. \& Paramasivan, C. N. (2003). What is new in the diagnosis of tuberculosis? Part II: techniques for drug susceptibility testing. Indian J Tuberc 50, 197-202.

Simboli, N., Takiff, H., McNerney, R., López, B., Martin, A., Palomino, J. C., Barrera, L. \& Ritacco, V. (2005). In-house phage amplification assay is a sound alternative for detecting rifampin-resistant Mycobacterium tuberculosis in low-resource settings. Antimicrob Agents Chemother 49, 425-427.

Solis, L. A., Shin, S. S., Han, L. L., Lanos, F., Stowell, M. \& Sloutsky, A. (2005). Validation of a rapid method for detection of Mycobacterium tuberculosis resistance to isoniazid and rifampin in Lima, Peru. Int J Tuberc Lung Dis 9, 760-764. 